\title{
On the stability of clathrate hydrates in comets 67P/Churyumov-Gerasimenko and 46P/Wirtanen (Research Note)
}

\author{
U. Marboeuf ${ }^{1}$, O. Mousis ${ }^{2}$, J.-M. Petit ${ }^{2}$, B. Schmitt ${ }^{1}$, A. L. Cochran ${ }^{3}$ and H. A. Weaver ${ }^{4}$ \\ ${ }^{1}$ Université Joseph Fourier, Laboratoire de Planétologie de Grenoble, CNRS INSU, France \\ e-mail: marboeuf@ujf-grenoble.fr \\ 2 Institut UTINAM, CNRS-UMR 6213, Observatoire de Besançon, BP 1615, 25010 Besançon Cedex, France \\ 3 McDonald Observatory, University of Texas, 1 University Station, C1402, Austin, TX 78712, USA \\ ${ }^{4}$ Space Department, Johns Hopkins University Applied Physics Laboratory, 11100 Johns Hopkins Road, Laurel, MD 20723-6099, \\ USA
}

Received 20 July 2010 / Accepted 1 November 2010

\section{ABSTRACT}

\begin{abstract}
Context. For several years, Jupiter-family comets have been the targets of spacecraft missions whose aims are to determine the comets' composition, structure, and physical properties. The Rosetta mission is currently flying towards comet 67P/Churyumov Gerasimenko for a rendezvous in August 2014 and comet 46P/Wirtanen is considered for a rendezvous in 2021 with the PriME (Primitive Material Explorer) mission, which is currently proposed to NASA.

Aims. Here we investigate the stability conditions of clathrate hydrates within the comets 67P/Churyumov-Gerasimenko and $46 \mathrm{P} /$ Wirtanen by considering an initial mixture of amorphous $\mathrm{H}_{2} \mathrm{O}$ with $\mathrm{CO}, \mathrm{CO}_{2}, \mathrm{CH}_{4}$, and $\mathrm{H}_{2} \mathrm{~S}$ in the nuclei.

Methods. We use a one-dimensional nucleus model, which considers an initially homogeneous sphere composed of a predefined porous mixture of ices and dust in specified proportions and describes heat transmission, gas diffusion, sublimation/recondensation of volatiles within the nucleus, water ice phase transition, dust release, and mantle formation.

Results. We show that stability conditions of multiple guest clathrates are permanently reached in the subsurface of both comets, and in a broader manner in the subsurface of all short period comets. The thickness of the stability zone of the clathrate slightly oscillates with time as a function of the heliocentric distance, but never vanishes. When comets approach perihelion, our calculations suggest that clathrate layers, which are located closer to the nucleus surface, may destabilize before amorphous ice is tranformed into crystalline ice.
\end{abstract}

Key words. comets: general - comets: individual: 67P/Churyumov-Gerasimenko - comets: individual: 46P/Wirtanen

\section{Introduction}

For several years, Jupiter-family comets (hereafter JFCs) have been the targets of spacecraft missions whose aims are to determine the comets' composition, structure, and physical properties. The Stardust mission has collected dust originating from Comet 81P/Wild 2 in January 2004 (Tsou et al. 2004), and the Rosetta mission is currently flying towards Comet 67P/Churyumov Gerasimenko for a rendezvous in August 2014 (Schulz et al. 2004). Moreover, Comet 46P/Wirtanen, which was the initial target of the Rosetta mission (Schulz \& Schwehm 1996), is again considered for a rendezvous in 2021 with the PriME (Primitive Material Explorer) mission, which is currently proposed to NASA as a Discovery mission (Cochran et al. 2010).

Marboeuf et al. (2010) recently proposed that $\mathrm{CO}$ and $\mathrm{CO}_{2}$ clathrate hydrates (hereafter clathrates) could exist within short period comets ${ }^{1}$ (hereafter SPCs). Clathrates are nonstoichiometric crystalline compounds, with hydrogen-bonded $\mathrm{H}_{2} \mathrm{O}$ molecules forming cages in which gas molecules are trapped individually by van der Waals interactions. At a given temperature, the stability of these cages is ensured as long as the gas phase pressure equals or exceeds the dissociation pressure of the considered clathrate. Using a thermodynamic model of JFCs including a mixture of $\mathrm{H}_{2} \mathrm{O}, \mathrm{CO}$, and $\mathrm{CO}_{2}$ ices,

\footnotetext{
${ }^{1}$ SPCs are divided into two categories: Halley type comets (periods between 20 and 200 years) and JFCs (period less than 20 years).
}

Marboeuf et al. (2010) showed that the stability zone of clathrates in these comets is restricted to a small part of the nucleus. They also found that the stability zone of clathrates could almost disappear at perihelion in Halley-type comets.

Using a mixture of gases, Mousis \& Schmitt (2008) showed that the resulting clathrate is also a mixture of volatiles whose equilibrium pressure is somewhere between that of the individual gases. In the present work, we consider a larger and more representative set of volatiles $\left(\mathrm{CO}, \mathrm{CO}_{2}, \mathrm{CH}_{4}\right.$, and $\left.\mathrm{H}_{2} \mathrm{~S}\right)$ that might exist in the nuclei compared to the one ( $\mathrm{CO}$ and $\mathrm{CO}_{2}$ ) initially adopted by Marboeuf et al. (2010). We then investigate the formation conditions of clathrates within the two JFCs 67P/Churyumov-Gerasimenko (hereafter 67PCG) and 46P/Wirtanen (hereafter 46PW) and show that irrespective of the latitude of the considered area, a permanent layer of multiple guest clathrates is likely to form in the subsurfaces of the two comets, and in a broader manner, in the subsurfaces of all SPCs. This suggests new directions of investigation for comet nucleus modelling which will be addressed in a forthcoming paper.

\section{Nucleus model}

The nucleus model employed in this work is based on the one-dimensional model developed by Marboeuf (2008) and Marboeuf et al. (2009). The model considers an initially homogeneous sphere composed of a predefined porous mixture of 
ices and dust in specified proportions. It describes heat transmission, gas diffusion, sublimation/recondensation of volatiles within the nucleus, water ice phase transition, dust release, and mantle formation. Water ice can be initially amorphous or crystalline, depending on the formation location of the body in the primitive nebula. When the ice is initially amorphous, a fraction of the volatiles is trapped in the water ice matrix and the remaining fraction forms pure condensates in the pores. When heated, the fraction of volatiles condensed in the pores sublimates first, and then the other fraction trapped within the matrix is released during the transition from amorphous to crystalline water ice. The released gas diffuses throughout the porous matrix by tortuous capillary tubes and eventually escapes from the object. The crystallization occurs on a temperature-dependant timescale $\tau(T)=9.54 \times 10^{-14} \mathrm{e}^{\frac{5370}{T}}$, where $T$ is the temperature (K) (Schmitt et al. 1989). Hence crystallization becomes efficient and effectively occurs at temperatures $\sim 110 \mathrm{~K}$.

These physical processes are described by the equation of diffusion of heat and mass. The heat diffusion throughout the nucleus is described by the energy conservation equation:

$$
\rho c \frac{\partial T}{\partial t}=\nabla \cdot\left(K \frac{\partial T}{\partial r}\right)-\sum_{x} H_{x}\left(Q_{x}+Q_{x}^{\mathrm{cr}}\right)+Y_{\mathrm{cr}} \quad\left(\mathrm{J} \mathrm{m}^{-3} \mathrm{~s}^{-1}\right)
$$

$$
\text { with } \rho c=\sum_{l} \rho^{l} c^{l}=\sum_{x} \rho_{x}^{i} c_{x}^{i}+\rho^{d} c^{d} \quad\left(\mathrm{~J} \mathrm{~m}^{-3} \mathrm{~K}^{-1}\right) \text {. }
$$

Here $t$ is the time (s), $K$ the heat conduction coefficient $\left(\mathrm{J} \mathrm{s}^{-1} \mathrm{~m}^{-1} \mathrm{~K}^{-1}\right)$ of the porous matrix, whose formula is based on geometric assumptions (Espinasse et al. 1993; Orosei et al. 1999), $r$ the distance (m) from the centre of the nucleus, $\rho^{l}$ the density $\left(\mathrm{kg} \mathrm{m}^{-3}\right)$ and $c^{l}$ the specific heat capacity $\left(\mathrm{J} \mathrm{kg}^{-1} \mathrm{~K}^{-1}\right)$ of the solid component $l$ in the comet nucleus $(l=d$ for dust or $i$ for pure ices of elements $x) . H_{x}$ is the molar latent heat of sublimation of ice $x\left(\mathrm{~J} \mathrm{~mol}^{-1}\right)$ and $Q_{x}^{\text {cr }}\left(\mathrm{mol} \mathrm{m}^{-3} \mathrm{~s}^{-1}\right)$ and $Y_{\mathrm{cr}}$ are the rate of moles of gas $x$ and the power per unit volume released during the crystallization process of amorphous water ice respectively (Espinasse et al. 1991; Orosei et al. 1999). $Q_{x}$ is the rate of volatile molecule $x\left(\mathrm{~mol} \mathrm{~m} \mathrm{~m}^{-3} \mathrm{~s}^{-1}\right)$ that sublimates/condenses in the pores of the matrix, and is given by the gas diffusion equation that describes the diffusion of gas through the porous matrix for each molecule $x$ :

$$
\frac{\partial \rho_{x}^{\mathrm{g}}}{\partial t}=\nabla \cdot\left(G_{x} \frac{\partial P_{x}}{\partial r}\right)+Q_{x}^{\mathrm{cr}}+Q_{x} \quad\left(\mathrm{~mol} \mathrm{~m}^{-3} \mathrm{~s}^{-1}\right),
$$

where $\rho_{x}^{\mathrm{g}}$ is the molar density of gas $x\left(\mathrm{~mol} \mathrm{~m}^{-3}\right), P_{x}$ its partial pressure $(\mathrm{Pa})$ and $G_{x}$ its diffusion coefficient $\left(\mathrm{mol} \mathrm{m}{ }^{-1} \mathrm{~s}^{-1} \mathrm{~Pa}^{-1}\right)$. The flux of gas diffusing through the porous matrix can be either a free molecular flow or a viscous flow, because the mechanism governing the diffusion of volatiles is selected according to the mean free path of molecules (Prialnik et al. 2004).

At the surface, the local temperature is given by a thermal balance between the adsorbed solar energy, the thermal emission, the heat diffusion towards the interior and the energy of sublimation of the existing ices (Orosei et al. 1999). Note that at each time step the porosity and the radius of pores are recomputed for each layer, taking into account the density variations of the solid phase.

\section{Thermodynamic parameters and initial composition}

At the beginning of the computation, the initial water ice of our nucleus is assumed to be amorphous (Taylor 1992; Kouchi et al. 1994). The JFCs come probably from the Kuiper Belt
Table 1. Physical parameters of the nuclei at the beginning of the computation.

\begin{tabular}{lcc}
\hline \hline Parameter & 67PCG & 46PW \\
\hline Orbital & 3.511 & 3.099 \\
Semimajor axis (AU) & 0.632 & 0.657 \\
Eccentricity & $2^{a}$ & $0.7^{b}$ \\
Radius (km) & $12.3^{a}$ & 6 \\
Rotational period (h) & \multicolumn{2}{c}{$0 \& 75$}
\end{tabular}

Molecular composition

$J_{x}$ (volatile $\mathrm{X} / \mathrm{H}_{2} \mathrm{O}$ molar ratio):

$J_{\mathrm{CO}}$ in pores (in amorphous ice)

$J_{\mathrm{CO}_{2}}$ in pores (in amorphous ice)

$J_{\mathrm{CH}_{4}}$ in pores (in amorphous ice)

$J_{\mathrm{H}_{2} \mathrm{~S}}$ in pores (in amorphous ice)

\author{
$11 \%(4 \%)$ \\ $2.5 \%(2.5 \%)$ \\ $1.5 \%(0.5 \%)$ \\ $1 \%(1 \%)$
}

Notes. ${ }^{(a)}$ Kossacki \& Szutowicz (2006), ${ }^{(b)}$ Kidger (2003).

(Lowry et al. 2008), which is populated by objects likely made from amorphous ice. Kouchi et al. (1994) showed that temperatures lower than $\sim 110 \mathrm{~K}$, corresponding to heliocentric distances greater than $\sim 12$ AU in the solar nebula would preserve the amorphous ice initially formed in the ISM. Below $\sim 12 \mathrm{AU}$, the disk temperature exceeded $\sim 110 \mathrm{~K}$, resulting in the crystallization of the amorphous grains embedded in that part of the nebula. In addition to water ice, the model takes into account the solid phases of $\mathrm{CO}, \mathrm{CO}_{2}, \mathrm{CH}_{4}$, and $\mathrm{H}_{2} \mathrm{~S}$. These molecules are among the most abundant volatile species (production rates relative to water greater than $1 \%$ ) observed in cometary nuclei (Bockelée-Morvan et al. 2004) and are susceptible to form clathrates at low-pressure conditions (Lunine \& Stevenson 1985). Other molecules such as $\mathrm{H}_{2} \mathrm{CO}, \mathrm{CH}_{3} \mathrm{OH}$, and $\mathrm{NH}_{3}$, which are also abundant in comets, have not been considered in this study because there are no experimental data concerning the equilibrium of $\mathrm{H}_{2} \mathrm{CO}$ and $\mathrm{CH}_{3} \mathrm{OH}$ clathrates (Fray \& Schmitt 2009; Marboeuf et al. 2008; Mousis et al. 2009), while $\mathrm{NH}_{3}$ does not form clathrates (Lunine \& Stevenson 1985). Table 1 gives the $\mathrm{X} / \mathrm{H}_{2} \mathrm{O}\left(J_{\mathrm{X}}\right)$ mole fractions relative to water (with $\mathrm{X}=\mathrm{CO}, \mathrm{CO}_{2}, \mathrm{CH}_{4}$ or $\mathrm{H}_{2} \mathrm{~S}$ ) of the volatiles initially present in the nucleus, either condensed in the porous network (first number), or trapped in the amorphous matrix (second number, in parenthesis). The latter depends on the environment temperature, the molecule (equilibrium pressure, size, polarizability) and its initial abundance in the molecular cloud (Kouchi et al. 1994; Bar-Nun et al. 2007), which leads to very large differences among the trapping efficiencies of various gases (Bar-Nun et al. 2007). Schmitt et al. (1989) showed that amorphous ice can trap other volatiles only up to $8 \%$ in mole of water. Unfortunately, there are currently no experimental data that give the relative proportions of volatiles trapped in amorphous ice and in porous network. We therefore choose plausible arbitrary initial concentrations, considering the initial abundances and equilibrium pressure of volatiles. Changing the concentrations by a factor of a few only speeds up or delays the progression of the crystalization and sublimation fronts, but not the actual temperature of the sublimation interface and the vapor pressure in the pores. In other words, this does not change the overall effects described here. The values of $J_{\mathrm{X}}$ are consistent with the observations in cometary comae of molecular species that are directly released from the nucleus (Bockelée-Morvan et al. 2004).

The vapour pressures of the pure condensates are obtained from Fray \& Schmitt (2009) and the amorphous to crystalline 
Table 2. Parameters of the equilibrium curves of the considered single guest clathrates.

\begin{tabular}{lccc}
\hline \hline Molecule & $A$ & $B$ & Reference \\
\hline $\mathrm{CO}$ & -1685.54 & 10.9946 & Hersant et al. (2004) \\
$\mathrm{CO}_{2}$ & -2663.17 & 12.3069 & Marboeuf et al. (2010) \\
$\mathrm{CH}_{4}$ & -2161.81 & 11.1249 & Hersant et al. (2004) \\
$\mathrm{H}_{2} \mathrm{~S}$ & -3111.02 & 11.3801 & Hersant et al. (2004) \\
\hline
\end{tabular}

Notes. $A$ is in $\mathrm{K}$ and $B$ is dimensionless.

ice phase transition is postulated exothermic ${ }^{2}$ and irreversible. Thermodynamic quantities characterizing other materials derive from Marboeuf et al. (2010). Table 1 summarizes the orbital parameters adopted in our study for comets 67PCG and 46PW.

The presence of several volatile compounds in the gas phase of the porous network can generate the formation of a multiple guest clathrate (hereafter MG clathrate) whose equilibrium pressure varies as a function of the gas phase composition and the temperature. The equilibrium pressure of the MG clathrate $P_{\mathrm{c}}$ is given by (Lipenkov and Istomin 2001; Hand et al. 2006)

$P_{\mathrm{c}}=\left(\sum \frac{y_{i}}{P_{i}^{\mathrm{c}}}\right)^{-1}$,

where $y_{i}$ is the mole fraction of the volatile $i$ in the gas phase and $P_{i}^{\mathrm{c}}$ the equilibrium pressure of the corresponding clathrate. Here we assume that the clathrate hydrate behaves as an ideally diluted solution and that the ratio of occupancies for cages is constant and the same for all guest molecules. The equilibrium pressures of the different single guest clathrates are modelled by $\log P_{i}^{\mathrm{c}}=A / T+B$, where $A$ and $B$ are constants (Miller 1961) whose values are given in Table 2 (with $P_{i}^{\mathrm{c}}$ in bars and $T$ in $\mathrm{K}$ ). When the gas pressure is higher than the equilibrium pressure of the MG clathrate, the ice and gas phases can combine to form the clathrate cages in the pores. Below the dissociation pressure, the cages become unstable, which leads to their dissociation, and only the ice and gas phase remain.

\section{Results}

We computed the thermodynamic evolution of comets 67PCG and 46PW as a function of time and for two angles of insolation. We find that the stability conditions of MG clathrates are permanently met in the subsurface, irrespective of the adopted angles of insolation and dust conductivities. Only the thickness of the clathrate layer is affected by these latter quantities. Figures 1 and 2 represent the evolution of the stratigraphies of comets 67PCG and $46 \mathrm{PW}$ over $70 \mathrm{yr}$ and $80 \mathrm{yr}$ timespans, respectively, for a latitude $\theta$ of $0^{\circ}$ (i.e., the maximum insolation angle) and a dust conductivity of $4 \mathrm{~W} \mathrm{~m}^{-1} \mathrm{~K}^{-1}$. The two figures show that the stability zone of the MG clathrate extends from the base of the amorphous-to-crystalline water ice phase transition layer up to the surface of the nuclei. This region grows slowly with time and also always contains the sublimation interfaces of $\mathrm{H}_{2} \mathrm{~S}$ and $\mathrm{CO}_{2}$ pure ices. In both comets, the gas phase, from which the MG clathrate can be formed, is dominated by $\mathrm{CO}(\sim 95 \%)$ and $\mathrm{CH}_{4}(\sim 5 \%)$ and contains small fractions of $\mathrm{H}_{2} \mathrm{~S}(\sim 0.1 \%)$ and $\mathrm{CO}_{2}(\sim 0.05 \%)$. When the comet approaches perihelion for the

\footnotetext{
2 It has been shown by Kouchi \& Sirono (2001) that crystallization of amorphous mixtures made of water and some other volatiles can become endothermic.
}

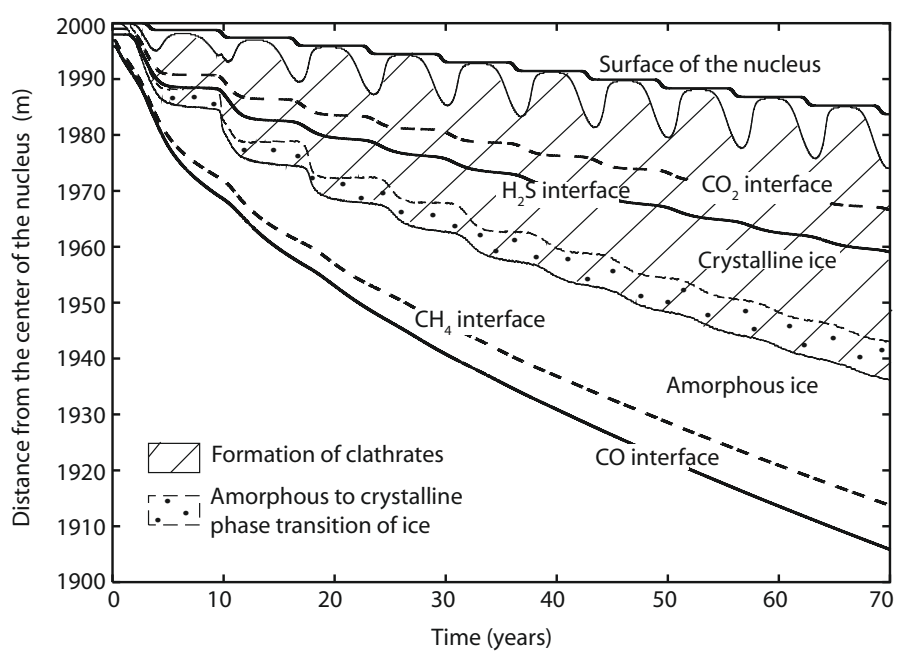

Fig. 1. Stratigraphy of the nucleus 67PCG as a function of time and for $\theta=0^{\circ}$. The lines represent the surface and the minimum depths at which solid $\mathrm{CO}_{2}$ and $\mathrm{CH}_{4}$ (dashed lines), $\mathrm{H}_{2} \mathrm{~S}$ and $\mathrm{CO}$ (bold solid lines) exist. The dotted area corresponds to the zone where the amorphous-tocrystalline water ice phase transition occurs and the dashed area to the zone of the MG clathrate stability.

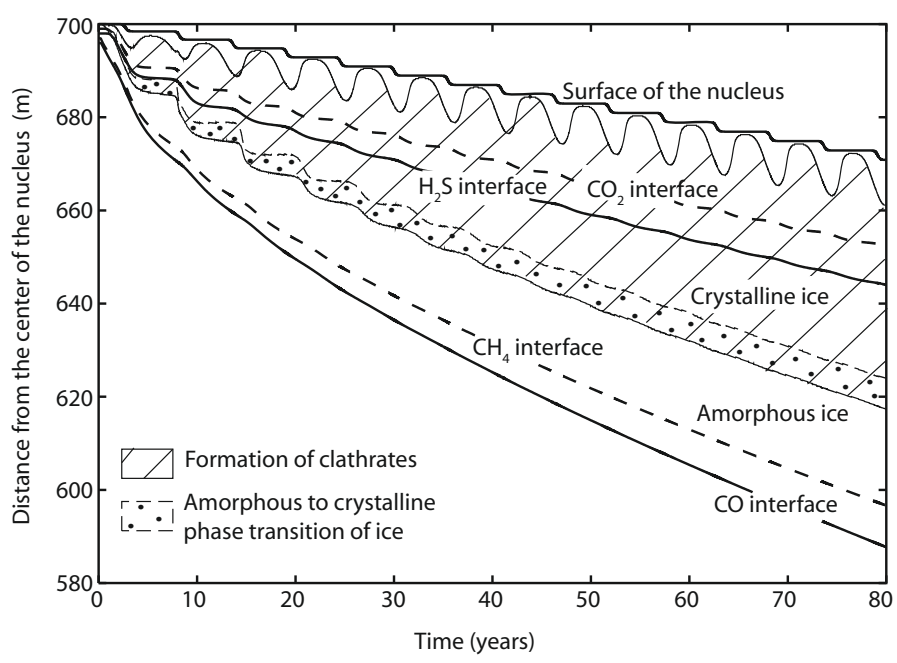

Fig. 2. Same as in Fig. 1, but for 46PW.

first time, the amorphous layers start to crystallize and $\mathrm{CO}, \mathrm{CO}_{2}$, $\mathrm{CH}_{4}$, and $\mathrm{H}_{2} \mathrm{~S}$ released in the pores can be enclathrated by the crystalline water ice available on their surface because the gas pressure (a few hundreds of $\mathrm{Pa}$ ) is greater than the clathrate equilibrium pressure (order of $1 \mathrm{~Pa}$ ).

As shown in Figs. 1 and 2, the thickness of the stability zone of the MG clathrate slightly oscillates with time as a function of the heliocentric distance, but never vanishes. In particular, when approaching the Sun, about the top $10 \mathrm{~m}$ of the clathrate layer destabilize and, inversely, become stable again as the nucleus cools. The destabilization of these top layers is caused by an increase of the local temperature at perihelion, which makes the MG clathrate equilibrium pressure rises above the gas pressure in the pores. When the comet moves away from perihelion, the layer cooling decreases the equilibrium pressure of the $\mathrm{MG}$ clathrate below the gas pressure in the pores, favouring again the possible formation of MG clathrate layers close to the surface, provided that there is enough available crystalline water ice. Note that at each perihelion passage, at least 1 to $2 \mathrm{~m}$ of new clathrate layer would be destabilized for the first time in 
subsurface, i.e. the gas pressure becomes lower than the clathrate equilibrium pressure. As an example, a region located in the middle of this oscillation zone has a gas pressure above that of clathrate equilibrium during more than three years for each orbit of Comet 67P/Churyumov-Gerasimenko.

It is interesting to note that the top clathrate layers, which are closer to the nucleus surface, are heated and destabilized when the comets approaches perihelion before the amorphous ice will be transformed into crystalline ice. We also performed tests with lower abundances of $\mathrm{CH}_{4}$ (4 times lower) and $\mathrm{H}_{2} \mathrm{~S}$ (20 times lower) to the lowest values in Bockelée-Morvan et al. (2004), and our results show that the stability area of clathrates varies only by a few percent.

\section{Discussion}

Our calculations show that assuming a plausible set of volatile abundances and irrespective of the adopted thermodynamic parameters of the nuclei, JFCs possess a region where clathrates would be stable, extending from the amorphous-to-crystalline water ice phase transition interface up to the surface. Additional calculations lead to the same results for Halley-type comets and then for all SPCs. The main improvement over Marboeuf et al. (2010) is the use of two additional volatiles $\left(\mathrm{CH}_{4}\right.$ and $\left.\mathrm{H}_{2} \mathrm{~S}\right)$, which increases the stability of $\mathrm{MG}$ clathrates. This allows the trapping of $\mathrm{CO}$ and $\mathrm{CO}_{2}$ at higher temperatures than if they were alone. Thus, clathrates containing $\mathrm{CO}$ and $\mathrm{CO}_{2}$ are permanently stable in much larger regions than shown in Marboeuf et al. (2010).

The permanent stability of clathrates in the subsurface of JFCs and SPCs raises two important questions. The first concerns the kinetics of clathrate formation. Do the various gases spend enough time inside the pores before they are outgassed at the surface to actually be trapped in clathrate cages? A rough estimate of this possibility for SPCs can be given with the following argument. The rate of formation/dissociation of clathrates in their stable/instable regions is mainly the function of the kinetics constant $\lambda^{\mathrm{cl}}$, the ice surface to volume ratio $A_{\mathrm{s}}$, and the difference between the gas pressure and the clathrate equilibrium pressure $\Delta P$, and can be writen as $\lambda^{\mathrm{cl}} A_{\mathrm{s}} \Delta P$ (Kim et al. 1987; Englezos et al. 1987; Schmitt 1986; Sun \& Mohanty 2006). On average, one has $\lambda^{\mathrm{cl}} \sim 5 \times 10^{-12} \mathrm{~mol} \mathrm{~m}^{-2} \mathrm{~Pa}^{-1} \mathrm{~s}^{-1}$ (Englezos et al. 1987), $A_{\mathrm{s}} \sim 2 \times 10^{4}$ in our model and $\Delta P \simeq 10-100 \mathrm{~Pa}$ in most of the region of stability of clathrates. Integrating the formation rate over the thickness of the clathrate stability layer ( $\sim 40 \mathrm{~m}$ as seen in Figs. 1 and 2$)$ we get a formation rate per unit surface of $4-40 \times 10^{-5} \mathrm{~mol} \mathrm{~m}^{-2} \mathrm{~s}^{-1}$. This is to be compared to the typical gas production rate at the surface of the nucleus of $0.8-4 \times 10^{-4} \mathrm{~mol} \mathrm{~m}^{-2} \mathrm{~s}^{-1}$. Hence the clathrate formation rate is similar to the classical degasing. At the same time, the amount of volatiles present in gas form per unit area of nucleus surface is $\sim 4 \mathrm{~mol} \mathrm{~m}^{-2}$. Hence the gaseous reservoir is only capable of supplying material for clathration for a few hours before there is a strong competition between clathration and degasing. Unfortunately, a precise evaluation of this effect can only be done with a fully self-consistent model.
This leads to the second question concerning the thermodynamics of the reactions. The present work is based on a comparison between the equilibrium pressure of clathrates and that of the gaseous phase in the porous network. In our model of a comet nucleus no clathrate forms (and thus decomposes) within the porous network. The influence of clathrate formation/dissociation on the internal temperature of the nucleus, pressure of the gaseous phase and gas production of SPCs is therefore not accounted for. We expect the temperature and gas pressure to be different in a self-consistent model, but we reckon this should not modify our main conclusions on the stability regions because the gas pressure is much higher than the clathrate equilibrium pressure. As for gas production, again only a fully self-consistent model can answer this question. Developping such a model accounting for the kinetics and thermodynamics of the formation/dissociation of clathrates is the subject of a forthcoming paper.

\section{References}

Bar-Nun, A., Herman, G., Laufer, D., \& Rappaport, M. L. 1985, Icarus, 63, 317 Bar-Nun, A., Notesco, G., \& Owen, T. 2007, Icarus, 190, 655

Bockelée-Morvan, D., Crovisier, J., Mumma, M. J., \& Weaver, H. A. 2004, Comets II, 391

Cochran, A. L., et al. 2010, BAAS, submitted

Delsemme, A. H., \& Miller, D. C. 1971, Planet. Space Sci., 19, 1229

Englezos, P., Kalogerakis, N., Dholabhai, P. D., \& Bishnoi, P. R. 1987, Chem. Eng. Sci., 42, 2647

Espinasse, S., Klinger, J., Ritz, C., \& Schmitt, B. 1991, Icarus, 92, 350

Espinasse, S., Coradini, A., Capria, M. T., et al. 1993, Planet. Space Sci., 41, 409 Fray, N., \& Schmitt, B. 2009, Planet. Space Sci., 57, 2053

Hand, D. P., Chyba, C. F., Carlson, R. W., \& Cooper, J. F. 2006, AstroBiology, 6,463

Hersant, F., Gautier, D., \& Lunine, J. I. 2004, Planet. Space Sci., 52, 623

Hudson, R. L., \& Donn, B. 1991, Icarus, 94, 326

Kidger, M. R. 2003, A\&A, 408, 767

Kim, H. C., Bishnoi, P. R., Heidemann, R. A., \& Rizvi, S. S. H. 1987, Chem. Eng. Sci., 42, 1645

Kossacki, K. J., \& Szutowicz, S. 2006, Planet. Space Sci., 54, 15

Kouchi, A., \& Sirono, S.-i. 2001, Geophys. Res. Lett., 28, 827

Kouchi, A., Yamamoto, T., Kozasa, T., Kuroda, T., \& Greenberg, J. M. 1994, A\&A, 290, 1009

Lipenkov, V. Y., \& Istomin, V. A., 2001, Mater. Glyatsiol. Issled, 91, 1 Lowry, S., Fitzsimmons, A., Lamy, P., \& Weissman, P. 2008, The Solar System Beyond Neptune, 397

Lunine, J. I., \& Stevenson, D. J. 1985, ApJS, 58, 493

Marboeuf U., 2008, Ph.D. Thesis, Univ. de Franche-Comté

Marboeuf, U., Mousis, O., Ehrenreich, D., et al. 2008, ApJ, 681, 1624

Marboeuf, U., Petit, J.-M., \& Mousis, O. 2009, MNRAS, 397, L74

Marboeuf, U., Mousis, O., Petit, J.-M., \& Schmitt, B. 2010, ApJ, 708, 812

Miller, S. L. 1961, Proceedings of the National Academy of Science, 47, 1798

Mousis, O., \& Schmitt, B. 2008, ApJ, 677, L67

Mousis, O., Lumine, J. I., Thomas, C., et al. 2009, ApJ, 691, 1780

Orosei, R., Capaccioni, F., Capria, M. T., et al. 1999, Planet. Space Sci., 47, 839 Prialnik, D., Benkhoff, J., \& Podolak, M. 2004, Comets II, 359

Schmitt B., 1986, Ph.D. Thesis, Univ. Joseph Fourier

Schulz, R., \& Schwehm, G. 1996, P\&SS, 44, 619

Schulz, R., Stüwe, J. A., \& Boehnhardt, H. 2004, A\&A, 422, L19

Schmitt, B., Espinasse, S., Grim, R. J. A., Greenberg, J. M., \& Klinger, J. 1989, Phys. Mech. Comet. Mat., 302, 65

Sun, X., \& Mohanty, K. K. 2006, Chem. Eng. Sci., 61, 3476

Taylor, S. R. 1992, Solar system evolution: a new perspective. An inquiry into the chemical composition, origin, and evolution of the solar system (Cambridge, New York: Cambridge University Press)

Tsou, P., Brownlee, D. E., Anderson, J. D., et al. 2004, JGRE, 109, 12 